\title{
DID YOU SEE THAT? A STUDY OF CHANGE BLINDNESS
}

\author{
Tara Smyser, John D. Lee, \\ Joshua Hoffman, Robert Betts \\ Department of Industrial Engineering \\ University of Iowa \\ 3131 Seamans Center \\ Iowa City, IA 52242-1527 \\ E-mail: tschisse@engineering.uiowa.edu
}

\section{OBJECTIVES}

Increasing concern has been expressed regarding the safety implications of drivers attempting to use in-vehicle devices other than cellular phones while driving. To address this issue, the effect of cognitive load on visual attention merits investigation. Strayer and Johnston (2001) found invehicle system use, specifically cell-phone use, disrupts performance by diverting attention to an engaging cognitive context other than the one immediately associated with driving. Recarte and Nunes (2000) demonstrated that scanning patterns and visual attention can be disrupted by cognitive load. In-vehicle systems can increase the cognitive load of the driver, making it more difficult for the driver to direct attention to the visual scene. The change blindness phenomenon may be useful way to investigate how cognitive load affects attention. Change blindness is the inability to detect scene change when the change occurs in conjunction with an action such as a blink (O'Regan, Deubel, Clark, and Rensink, 2000), eye movement (Wallis and Bulthoff, 2000), or an image that masks the onset of the change (Simons and Levin, 1998). The aim of this experiment was to study the effect of cognitive loading on individuals' ability to detect change in their visual environment using a speech-based email task.

\section{METHODS}

Twenty participants completed a series of five conditions. In two of these conditions the participants did just one task: either the e-mail task or the visual search task. In the other three conditions participants completed the e-mail and the visual search tasks concurrently. In one email task condition the speech recognition system worked perfectly. In a second condition, speech recognition errors caused the wrong menu item to be selected. In a third condition, speech recognition errors caused the user to be displaced to the wrong menu. In three of the visual search task conditions participants were asked to identify changes that occurred in visual scenes, using the Rensink, et al. (1997) flicker paradigm while navigating a speech-based e-mail system. The visual task presented a series of four displays. These were: an unaltered image (300ms), a gray screen $(1150 \mathrm{~ms})$, a second image $(300 \mathrm{~ms})$, and a gray screen $(1150 \mathrm{~ms})$. The second image was either the unaltered image or an image altered by the addition or removal of an element. The remaining conditions for both the e-mail task and visual search task were labeled as baseline. The baseline measurements were used to evaluate the effects of cognitive load on detection of scene changes. 


\section{RESULTS}

Analyses showed that detection of scene changes took significantly longer when participants were cognitively loaded with the e-mail task (mean 5.05) compared to when they were not (mean 4.35), $\mathrm{F}(3,67)=11.13, \mathrm{p}<0.0001$. Analyses also showed participants took significantly longer to determine that the scene had not changed (mean 5.99) than to detect a change had occurred (mean 3.72), $\mathrm{F}(2,67)=271.95, \mathrm{p}<0.0001$. Scene detection accuracy was significantly reduced when participants were cognitively loaded with the e-mail task, $F(3,67)=5.47$, p0.0010. Speech recognition errors introduced by the researcher had little effect on times to determine scene changes as well as time to detect meaningful and non-meaningful scene changes.

\section{CONCLUSION}

The results of this study demonstrate that change detection is sensitive to cognitive load and that endogenous control of visual attention may have been affected by the introduction of the e-mail system. It also shows that the paradigm of scenes with and without changes seems to be a promising and sensitive tool for measuring the effects of cognitive load on an individual's ability to detect change.

\section{REFERENCES}

O'Regan, J.K., H. Deubel, J.J. Clark, \& R.A. Rensink. (2000). Picture changes during blinks: Looking without seeing and seeing without looking. Visual Cognition 7(1-3), 191-211.

Recarte, M.A. \& Nunes, L.M. (2000). Effects of verbal and spatial imagery tasks to eye fixations while driving. Journal of Experimental Psychology: Applied,6, 31-43

Rensink, R.A., Oregan, J.K., \& Clark, J.J. (1997). To see or not see: The need for attention to perceive changes in scenes. Psychological Sciences, 8, 368-373

Simons, D.J. and D.T. Levin. (1998). Failure to detect changes to people during a real-world interaction. Psychonomic Bulletin \& Review 5(4), 644-649.

Strayer, D.L. \& Johnston, W.A. (2001). Driven to distraction: Dual-task studies of simulated driving and conversing on a cellular phone. Psychological Science, 12, 462-466

Wallis, G. and H. Bulthoff. (2000). What's scene and not seen: Influences of movement and task upon what we see. Visual Cognition 7(1-3), 175-190. 